\title{
Neurosurgery resumption in Wuhan during the early post-epidemic period: what should we pay attention to?
}

\author{
Yiwei $\mathrm{Qi}^{1,2} \cdot$ Xiaojin Liu $^{1,2} \cdot$ Xueyan Wan ${ }^{1} \cdot$ Liang Lu $^{1,2} \cdot$ Feng Hu$^{1} \cdot$ Chunxia Qi ${ }^{1} \cdot$ Kai Zhao $^{1} \cdot$ Kai Shu $^{1} \cdot$ Ting Lei $^{1,2}$ (D)
}

Received: 18 August 2020 / Revised: 28 October 2020 / Accepted: 19 March 2021 / Published online: 10 April 2021

(c) The Author(s), under exclusive licence to Springer-Verlag GmbH Germany, part of Springer Nature 2021

\begin{abstract}
COVID-19 has spread globally, causing a pandemic and medical interruptions. As more countries control the epidemic, the resumption of work is imperative. However, asymptomatic carriers become the main source of infection. After several months of recovery, Wuhan had much experience with facing the challenge of work resumption. The purpose of this study was to investigate the safety of the resumption strategies, as well as the outcome of the resumption efforts, in the early postepidemic period. A retrospective study was conducted in patients admitted between April 8 and June 30 to the neurosurgery department of Tongji Hospital, Wuhan. The medical information, past medical history, COVID-19 tests, laboratory parameters, CT results, and management were reviewed and recorded. 768 patients were admitted to the neurosurgery department at Tongji Hospital, and none of them became new infections. Our department recovered to $70 \%$ efficiency one month after the resumption of work. Two patients were found to have asymptomatic infections in the outpatient department. Two patients who recovered from COVID-19 underwent the surgery without recurrence of COVID-19. Tumor patients accounted for more than $50 \%$ of the surgery patients in the early period. It is feasible and helpful to follow our strict admission algorithm in the early post-epidemic period, even though the challenges of asymptomatic infectors exist. Two COVID-19 tests in 3 days are suggested within the early period. Protective downgrades should be based on the testing of asymptomatic patients in the area. Recovered COVID-19 patients can undergo surgery without recurrence.
\end{abstract}

Keywords Neurosurgery · Post-epidemic period · Work and production Resumption · Admission algorithm · COVID-19

\section{Introduction}

Since coronavirus disease 2019 (COVID-19) was first reported, the virus has spread around the world and become a pandemic leading to medical interruptions. The number of patients with COVID-19 worldwide increases more than 100 000 per day, with $11,622,741$ infected and 538,172 deaths as of July 7 of 2020 [16]. Despite being the first country to be attacked by COVID-19, the People's Republic of China designed a series of urgent policies and strict lockdowns to

Ting Lei

tlei@tjh.tjmu.edu.cn

1 Department of Neurosurgery, Tongji Hospital, Tongji Medical College, Huazhong University of Science and Technology, Wuhan 430030, China

2 Department of Neurosurgery, Sino-German Neuro-Oncology Molecular Laboratory, Tongji Hospital, Tongji Medical College, Huazhong University of Science and Technology, Wuhan 430030, China halt the spread. On April 8, after 76 days of lockdown, people in Wuhan were allowed to return to work under COVID19 prevention guidance [10]. At present, the pandemic in China has been well controlled, and work and production have been resumed for two months.

Although recommendations regarding the handling of the pandemic have been provided, medical services have been seriously affected yet. Therefore, neurosurgeons all over the world have participated in hot topic discussions about how to resume surgery after the outbreak. However, a new challenge facing us is that patients with asymptomatic and mildly symptomatic infections are becoming potential sources of infection during post-epidemic recovery. Asymptomatic individuals do not know that they are infected and do not exhibit any specific symptoms. As the positive screening accuracy of throat swabs is no more than $50 \%[1,11]$, asymptomatic infected persons may be missed and admitted to the hospital, inadvertently triggering new infections. There is an underlying risk of contracting COVID-19 for people in the hospital during the early post-epidemic period. 
There is a general concern about asymptomatic individuals among patients hospitalized in a city such as Wuhan, which was seriously affected by COVID-19. It is vital to manage patients during the post-epidemic period. In addition, it was reported that patients with chronic diseases such as diabetes are more likely to become infected [2]. Theoretically, patients are more easily infected during transsphenoidal surgery [6]. However, there is a question of whether there are certain types of hospitalized patients or procedures in the neurosurgical department that are associated with a higher risk of infection during the post-epidemic period. The management algorithm to keep patients and medical staff safe and the ability of neurosurgery programs to recover from the outbreak remain unclear. To date, there have been no reports addressing these issues, so it is not clear what we should pay attention to.

After Wuhan had been in the post-epidemic period for three months, we conducted a retrospective study at our center from April 8 to June 30 to answer these questions. As the largest neurosurgical center in Wuhan city, our department currently completes 15 operations daily, and more than 20 patients are admitted on average per day. With more countries enteringthe post-epidemic period, we hope that the information presented from our research is meaningful.

\section{Materials and methods}

\section{Study design}

This was a retrospective study involving patients admitted to the neurosurgical department in Tongji Hospital, Wuhan, China. All patients were admitted to the hospital while following the COVID-19 management procedure between April 8 and June 30. Patients with incomplete clinical and radiological data were excluded.

This study was approved by the Ethical Committee of Tongji Hospital affiliated to Tongji Medical College of Huazhong University of Science and Technology. Patients were written informed consent has been waived by the Ethics Committee because of the retrospective and anonymous nature of the data.

\section{Patient management}

\section{Change in infrastructure}

We remodeled part of the existing rooms into new rooms, wards, and intensive-care units for isolation treatment based on past experience [12]. The specific change in the infrastructure can be seen in Fig. 1. The observation area had a special access entry and consisted of separate rooms. The removed area had several wards without connection.
Recovered patient area include several separate rooms for quarantine.

\section{Patient classification}

All patients were classified according to the sixth edition of the COVID-19 diagnosis and treatment plan issued by the National Health Commission.

\section{Patient' admission algorithm}

During the first stage (April 8 to May 25) patients admitted from the outpatient department were required to have their temperature taken and a routine blood test. The medical history of COVID-19 infection was also inquired. Additonally, all patients and the accompanying person had a pulmonary CT scan (€26.5), COVID-19 nucleic acid (€16.7), and an antibody test $(€ 10.1)$. Any patient with a positive nucleic acid test was transferred to the designated medical institution.

If a patient and his or her accompanying family had negative COVID-19 nucleic acid and antibody tests, they were allowed to be admitted; they waited for another test and pulmonary CT during 3 days of observation in the observation area. During the observation period, patients and their accompanying persons were not allowed to leave the room. When the second test and symptoms were negative, patients were transferred to the removed area. The observation area had its special access entry and was consisted of separate rooms. The removed area was divided into several wards without connection. Surgeons, nurses, and health-care workers in the observation area used appropriate personal protective equipment based on Level-II protective standards. Level-I protective standards were suggested for the removed area.

Patients who had a positive antibody test were required to have a second nucleic acid and antibody test before admission. Patients with two positive IgM antibody tests but negative nucleic acid tests were recorded and not allowed to be admitted. Patients with two positive $\mathrm{IgG}$ antibody tests but negative nucleic acid tests were admitted to the recovered patient area. Medical staff in the recovered patient area chose appropriate personal protective equipment based on Level-II protective standards.

If patients had a susceptible sign on pulmonary CT, a medical consultation before admission was required. They were allowed to wait in the observation area for three days observation followed by a second COVID-19 tests. If the second CT had suspicious signs, prolonged observation time and another CT were required.

Patients admitted from the emergency room had the same tests prior to surgery. After the operation, suspected patients were urgently admitted to the intensive care unit observation 
Inpatient Building

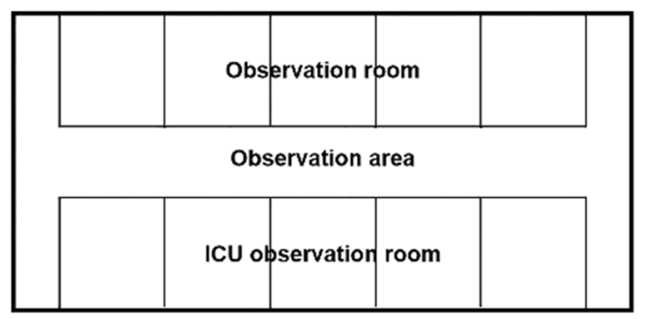

\begin{tabular}{|c|c|c|c|c|c|c|}
\hline $\begin{array}{l}\text { Recov- } \\
\text { ered } \\
\text { room }\end{array}$ & $\begin{array}{l}\text { Recov- } \\
\text { ered } \\
\text { room }\end{array}$ & $\begin{array}{l}\text { Alter- } \\
\text { able } \\
\text { room }\end{array}$ & $\begin{array}{l}\text { Genera } \\
\text { I room }\end{array}$ & $\begin{array}{l}\text { Genera } \\
\text { I room }\end{array}$ & $\begin{array}{l}\text { Genera } \\
\text { I room }\end{array}$ & $\begin{array}{l}\text { Genera } \\
\text { I room }\end{array}$ \\
\hline \multicolumn{2}{|c|}{ Recovered patient area } & \multicolumn{5}{|c|}{ Removed area } \\
\hline $\begin{array}{l}\text { Recov- } \\
\text { ered } \\
\text { room }\end{array}$ & $\begin{array}{c}\text { Recov- } \\
\text { ered } \\
\text { room }\end{array}$ & \multicolumn{5}{|c|}{ ICU } \\
\hline
\end{tabular}

Operation Building No. 1

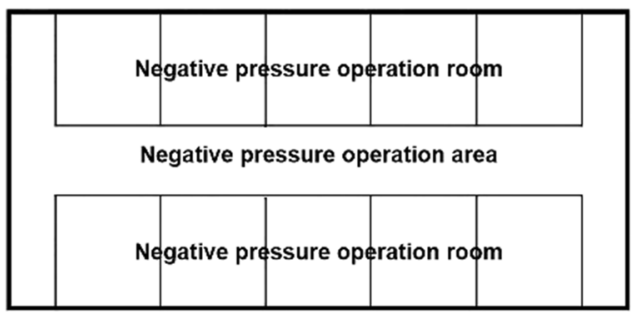

Fig. 1 The specific change in the infrastructure. The observation area and removed area are different wards which are not connected. Necessary wall partitions and protection have been added in different areas. The alterable room will be used as an emergency isolation room if

room and had nucleic acid and antibody tests for confirmation. It was necessary to recheck the pulmonary CT when suspicious symptoms occurred. The admission algorithm is presented in Fig. 2.

In the second stage (May 26 to June 11), the observation time was shortened to 1 day after the nucleic test. $11,000,000$ Wuhan people were tested. An epidemiological mass survey showed that only a very small number of asymptomatic infections occur. Pulmonary CT was gradually eliminated. Other procedures followed the same as those in the first stage.

During the third stage (June 12 to June 30), patients were allowed to have the first test for COVID-19 in a local hospital 7 days before admission. If the patient had their first test in a local hospital, the second COVID-19 tests was conducted at the outpatient department. Pulmonary $\mathrm{CT}$ was not performed. Other procedures remained the same.

\section{Operation management}

\section{Emergency operation}

Before the test results returned, patients underwent surgery in an isolation operation room that had a negative-pressure necessary. Different areas have special access. The Operation Building 1 has been remodeled for outpatient emergency surgery and surgery for COVID-19 patients

environment or an independent airflow system. Level-III protective standards were adopted in emergency operations. All medical supplies were fully prepared in the designated isolation room prior to surgery. During the operation, the medical staff, which included two surgeons and one nurse, were not allowed to leave of the operating room, and one specific circulating nurse was required. Routine methods were suggested to reduce operation time. Temperature, respiratory function, and blood pressure were carefully monitored. A special transfer channel from the operation room to the intensive care unit observation room was used.

\section{Elective surgery}

For a healthy patient, the elective surgery was performed in an ordinary operating room in a routine manner. However, for recovered patients, surgery was performed only if there were no symptoms of cough or fever, and the anesthesiologist and respiratory physician agreed to the surgery. A careful plan was made to shorten the operation time. The operation was also performed in an isolation operation room, as was the case for emergency operation. Level-III protective standards were adopted for use in recovered patients undergoing elective surgery. Level-II protective standards were in other elective surgery. The 
Fig. 2 The algorithm of hospital admission. + represents a positive test result.-represents a negative test result. ? represents suspicious signs on CT scan

\section{Admission alignment}

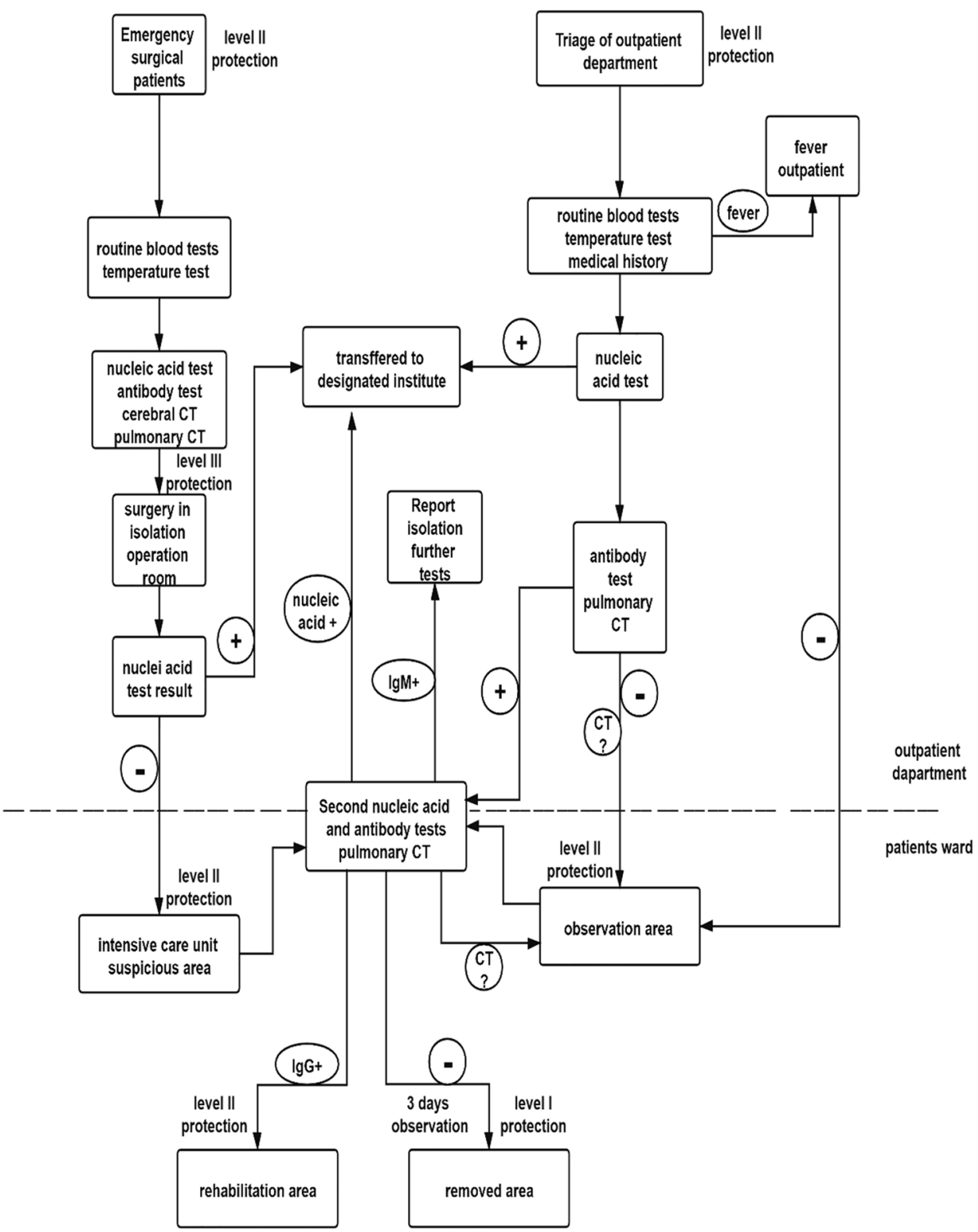

patient's blood pressure and respiratory function were closely monitored during the operation, as was blood coagulation function.

Myocardial enzymes, renal function, liver function, coagulation function, routine blood test, arterial oxygen saturation, and cerebral CT were monitored after both operations. Pulmonary CT was necessary once suspicious symptoms were found.

\section{Data collections}

Data of all patients, including sex, age, address information, medical history, comorbidities, pulmonary CT scans, COVID-19 nucleic and antibody tests, laboratory tests, inhospital procedures, activity of daily living assessed by Barthel Index, and complications, were extracted by two independent experienced data coordinators through Epidata 3.1 
from the electronic medical records. Phone surveys were conducted to determine whether the discharged patient had a new infection.

\section{Statistical analysis}

The measurement data were presented as the mean \pm standard deviation (SD). The comparison between the two groups was performed by independent student t-tests, for the normally distributed measurement data. Otherwise, Mann-Whitney U tests were applied. Enumeration data were summarized as frequency rates and percentages. Chi-squared tests or Fisher's exact tests were performed for enumeration data. Graphic and statistical analyses were conducted by GraphPad Prism or Microsoft Excel.

\section{Results}

Two asymptomatic patients identified in the outpatient department were transferred to the designated COVID-19 medical institute early April. Neither of them had any symptom or knew when they has been infected.Three patients had two positive IgM antibody tests, but negative nucleic acid tests in the outpatient department. The infections were recorded and the patients were asked to stay at home for further observation. A total of 768 patients were admitted to our center, and 712 patients underwent surgery from April 8 to 30 June. $11.33 \%$ of these patients came from high-risk areas. A total of 397 male patients and 371 female patients were admitted. The average age was $45.3 \pm 18.7$ years. $11.72 \%$ were younger than 18 years old, $66.93 \%$ were between 18 and 60 , and $21.35 \%$ were older than 60 . The median value of the Barthel Index was 80 . The composition of the disease was as follows: cerebrovascular disease and hemorrhage (CBDH) accounted for $18.36 \%$, trauma occupied $10.81 \%$, intracranial tumor representing $49.35 \%$, functional disease for $2.86 \%$, spinal tumors constituting $5.21 \%$, hydrocephalus taking up $5.60 \%$ and other diseases comprising $7.81 \%$ of all diseases. Among all the surgeries, transsphenoidal surgery accounted for $6.04 \%$, craniotomy for $88.35 \%$, and spinal surgery occupied $5.61 \%$. Emergency operations accounted for $15.92 \%$ of total operation numbers. The characteristic summary is shown in Fig. 3. Complex surgeries, including tumor resection, cerebrovascular reconstruction, and others, had a proportion of $77 \%$. The comparison curve of this year's surgical procedures and last year's procedures is shown in Fig. 4. A total of $9.80 \%$ of patients had a white cell count lower than $4 \times 10^{\wedge} 9 / \mathrm{L}$. Patients accounted for $17.65 \%$ of total patients had a white cell count higher than $10 \times 10^{9} / \mathrm{L}$. A total of $18.95 \%$ of patients had a lymphocyte count lower than $1.1 \times 10^{9} / \mathrm{L}$. Additionally, 37 patients $(4.82 \%)$ with an average age of 43.7 had suspicious ground-glass opacities on pulmonary CT. in the average age of 43.7. Two were recovered patients and others turned to not have COVID19. Among patients with suspicious signs on CT, $18.92 \%$ of patients came from the high-risk area. A comparison between the patients with suspicious COVID-19 signs on $\mathrm{CT}$ and CT normal patients is shown in Table 1. No patient had a lower WBC count and a suspicious CT. The complication rate was $10.77 \%$. The main complications were cerebral infarction, intracranial infection, cerebrospinal fluid leakage, epilepsy, and hydrocephalus.

Two recovered patients ( 1 male and 1 female) had two positive IgG antibody tests with negative nucleic acid tests and underwent the operation without recurrence. These two recovered patients both came from high-risk areas and were tumor patients aged 33 and 61 years. Case 1 was a female case. She was diagnosed with hydrocephalus after a tumor operation. She was brought to the hospital because of a headache on April 7. She underwent a ventriculoperitoneal shunt and had a history of diabetes. Case 2 was a male patient who was diagnosed with neurilemmoma of the spinal cord. The male patient underwent spinal surgery and had a history of cerebral infarction. Neither had COVID-19 symptoms before or after surgery.

There were no new infections among the hospitalized patients in our center. No medical staff members or persons accompanying the patients were newly infected from April 8 to June 30 .

\section{Discussion}

To our knowledge, this is the first report of what did the neurosurgical department look like and resumption strategies during the COVID-19 post-epidemic period in the first outbreak city, Wuhan. After several months of practice and recovery, we have gained much experience. We attempted to determine what we should pay more attention to in the early stage of recovery. Since COVID-19 is gradually being controlled in many countries and areas, asymptomatic and mildly symptomatic individuals replace those with typical and severe infections as potential sources of infection. There is widespread anxiety $[4,5]$ about the existence of asymptomatic carriers among hospitalized patients. There have been reports of clusters of hospitalized patient infections traced to asymptomatic infectors [1,14]. Although work and production resumption are required in many countries and areas, an increase in COVID-19 was reported after resumption [15]. It is vital to determine safe and efficient procedures and management alignment. Distinguishing asymptomatic infectors is necessary. In our retrospective study during the early postepidemic period, asymptomatic carriers were successfully found before admission and transferred to designated institutes to avoid new infections. A total of 768 patients were 
Fig. 3 a Patient number composing of male and female including surgery or not. $\mathbf{b}$ Disease composition (CVDH for cerebrovascular disease and hemorrhage) c Age distribution $\mathbf{d}$ Classification of surgical approaches

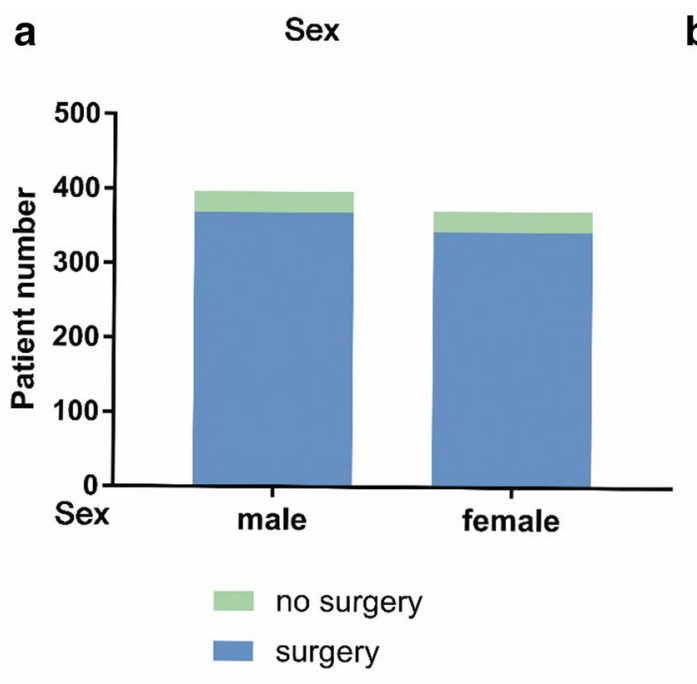

b
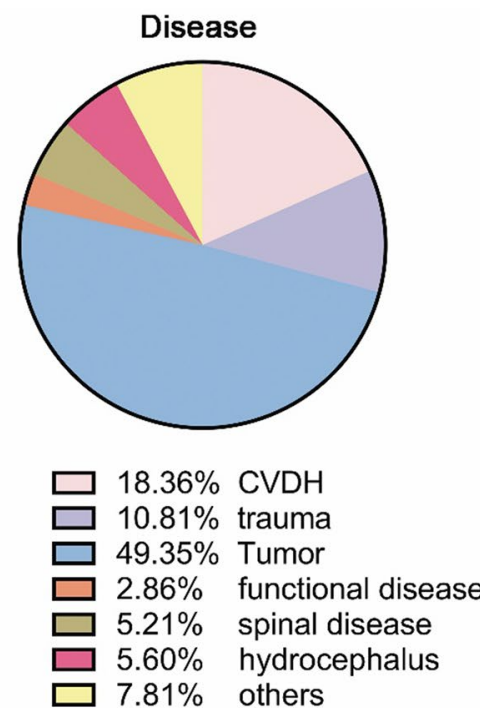

c

Age

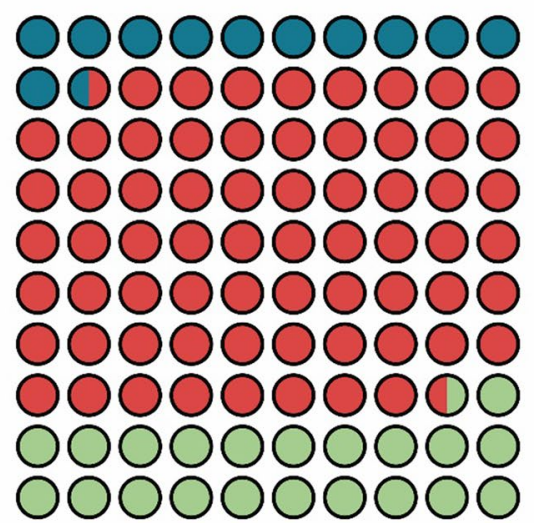

d Surgery

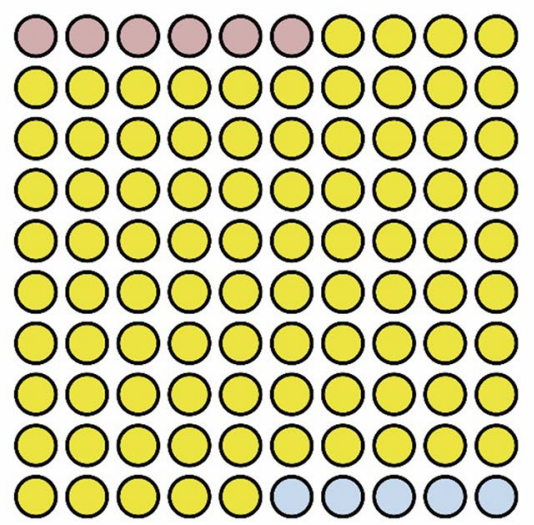

$6.04 \%$ transsphenoidal surgery

$88.76 \%$ craniotomy

$5.20 \%$ spinal surgery
Fig. 4 The comparison curve of this year's surgical procedures and last year's procedures

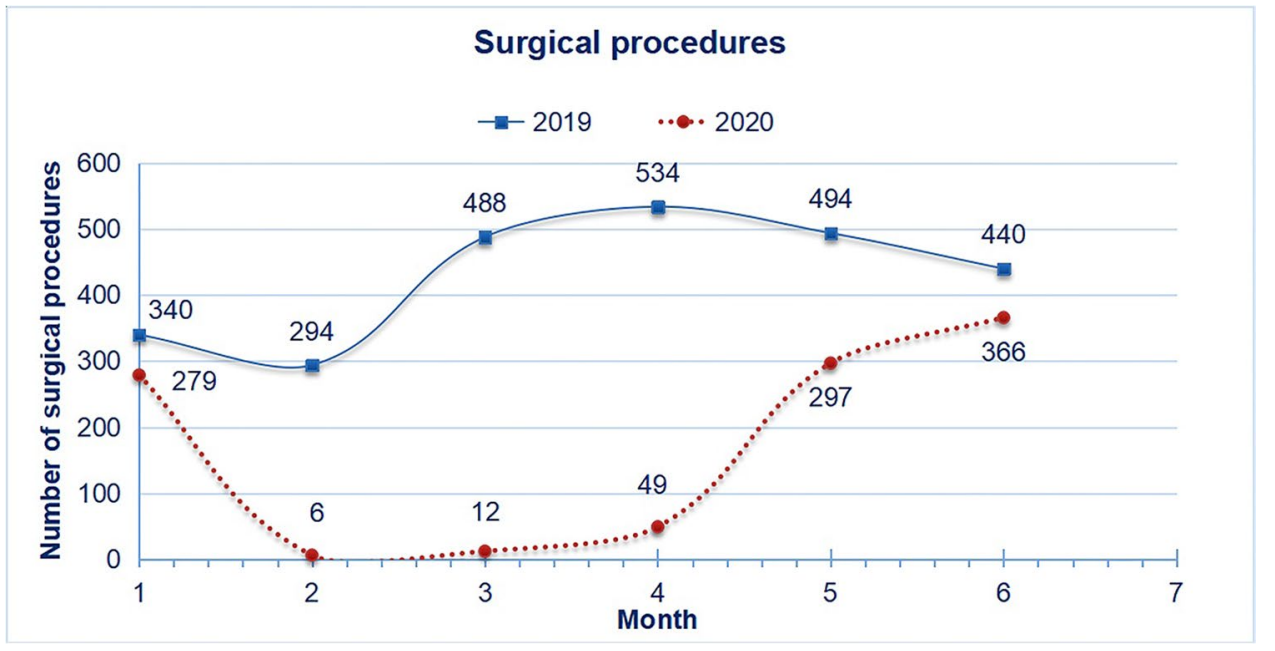


Table 1 Comparisons between patients with suspicious sign in CT and patients with normal CT

\begin{tabular}{|c|c|c|c|}
\hline & $\begin{array}{l}\text { Patients with } \\
\text { suspicious sign } \\
\text { in CT }\end{array}$ & $\begin{array}{l}\text { Patients } \\
\text { with normal } \\
\text { CT }\end{array}$ & P value ${ }^{a}$ \\
\hline Positive Nucleic acid test & 0 & 0 & \\
\hline $\begin{array}{l}\text { Positive IgG antibody } \\
\text { test }\end{array}$ & 2 & 0 & $<0.01$ \\
\hline Male/female & $20 / 17$ & $377 / 354$ & 0.87 \\
\hline $\operatorname{Age}(y$, mean, $[\mathrm{SD}])$ & $43.7 \pm 17.7$ & $45.4 \pm 18.9$ & 0.61 \\
\hline $0-18$ & 4 & 83 & \\
\hline $18-59$ & 27 & 492 & \\
\hline $60-79$ & 6 & 154 & \\
\hline$\geq 80$ & 0 & 2 & \\
\hline Area & & & 0.18 \\
\hline High risk area & 7 & 80 & \\
\hline Low risk area & 30 & 651 & \\
\hline Admission patterns & & & 0.82 \\
\hline emergency & 5 & 117 & \\
\hline General admission & 32 & 614 & \\
\hline \multicolumn{4}{|l|}{ Comorbidities } \\
\hline Diabetes & 14 & 120 & $<0.01 *$ \\
\hline Cardiovascular disease & 4 & 35 & 0.10 \\
\hline Malignancy & 7 & 86 & 0.19 \\
\hline Neurosurgical procedure & $36 / 37$ & $676 / 731$ & \\
\hline transsphenoidal & 2 & 41 & 0.96 \\
\hline craniotomy & 33 & 599 & 0.26 \\
\hline spinal surgery & 1 & 36 & 0.54 \\
\hline White blood cell count & & & $<0.01^{*}$ \\
\hline$<4$ & 0 & 57 & \\
\hline $4-10$ & 23 & 591 & \\
\hline$>10$ & 14 & 83 & \\
\hline Lymphocyte count & & & 0.06 \\
\hline$<1.1$ & 8 & 84 & \\
\hline$\geq 1.1$ & 29 & 647 & \\
\hline PT & $13.5 \pm 1.1$ & $13.4 \pm 1.0$ & 0.86 \\
\hline APTT & $38.6 \pm 3.8$ & $38.2 \pm 5.8$ & 0.67 \\
\hline Glucose & $6.0 \pm 2.0$ & $5.7 \pm 1.8$ & 0.46 \\
\hline
\end{tabular}

$C T$, computed tomography; $S D$, standard deviation

${ }^{\text {a }} \mathrm{P}$ values comparing between patients with suspicious sign in $\mathrm{CT}$ and patients with normal CT are from $\chi^{2}$ test, Fisher's exact test, $t$ test or Mann-Whitney U test

*Significant at $\mathrm{P}<0.05$

admitted to our centers, and no new infection was found. While following the admission algorithm, our neurosurgical center returned to $70 \%$ of normal efficiency in as soon as one month. These results showed that our recovery strategies were effective.

Susceptible populations are the focus of infectious disease prevention and control. Active detection of suspected patients is a good way to avoid nosocomial infections. For admission, laboratory physicians suggest that it is necessary to have two negative results [11]. In the early stage of resumption, it was not clear that patients could be transferred to the removed area until after three days of observation with no symptoms and a negative test. However, when the proportion of asymptomatic patients indicated by a large number of tests was low, the detection process was simplified, and shorter observation periods were allowed. In addition, some patients need more attention. It was reported that patients with diabetes had an increased risk [2] of becoming infected. One recovered COVID-19 patient with diabetes was carefully checked and identified in the outpatient department. Patients with chronic diseases such as diabetes tended to have suspicious signs of COVID-19 on CT in our study. Therefore, diabetic patients have a higher rate of asymptomatic infection, which deserves more attention. However, as previously reported, most asymptomatic cases had no remarkable laboratory findings, but lung opacities were present in half of the cases [7]. This was the same as in our results. Susceptible populations require highly sensitive tests. The sensitivity of pulmonary CT in infected patients was reported to be more than $90 \%$ [3]. Since the first stage of resumption is the most dangerous stage, the epidemic is prone to relapse. We need to perform high sensitivity testing in susceptible populations. Therefore, we consider the CT results as an important test required for admission [9] in the first stage. Both recovered patients were found through pulmonary CT before the antibody test, demonstrating the sensitivity of CT. However, it was still difficult to use CT to distinguish other infection patients from COVID-19 patients when both had similar signs, such as ground-glass opacities. Taking into account the radiation involved, we gradually reduced the use of pulmonary CT at the beginning of stage 2. Pulmonary CT was not used for epidemiological mass surveys.

What is the effect of COVID-19 on patients after the outbreak? Through the analysis of disease species, we found that patients bearing tumors composed a high proportion of hospitalized patients in the post-epidemic period because cancer patients are more eager to be treated. Hospitalized patients were sicker this year than last year. However, there was no significant difference between sexes. Patients aged between 18 and 60 years were the major group, but, overall, the patients were younger this year than the same period of the last year. The results may have to do with the fact that people in this age group have more responsibilities after work resumption. In addition, the mental health problems of patients also need to be paid enough attention.

The surgical approach is critical to neurosurgery. Recurrence presenting with meningoencephalitis has been reported [8]. As there was no definition for when recovered patients could undergo neurosurgery, we tried to establish one ourselves. Breath-holding tests, laboratory 
results, and symptoms were confirmed repeatedly before the operation to determine if the patient was suitable for surgery. It was our experience that preoperative lung function should be close to $90 \%$ of the normal level for the elective surgery. Mental health care was helpful for recovery. For pituitary tumor patients, transsphenoidal surgery close to the respiratory tract was considered dangerous during the COVID-19 pandemic [6]. In our center, 43 transsphenoidal approach surgeries were complete without new infections. Partial transsphenoidal surgery and craniotomy were performed by the same surgeon. Thus, the transsphenoidal approach did not increase the patient's COVID-19 infection rate during the post-epidemic period following rigorous management guidance. All neurosurgical operations are safe if guidelines are followed.

In our management procedures, we need to explain a few small details. Movement of individuals between the observation area and other areas was not allowed, because asymptomatic infectors can spread the virus through the environment [13].

\section{Conclusion}

While facing the challenge of asymptomatic infectors, it is feasible and helpful to follow our strict admission algorithm in the early post-epidemic period. Two COVID-19 tests in 3 days are of helpful and suggested during the early period. Protective downgrades should be based on the testing of asymptomatic patients in the area. Recovered patients underwent neurosurgery without the recurrence of COVID-19. Patients with suspicious signs of COVID-19 on CT and a history of chronic diseases (such as diabetes) should be carefully managed. We are pleased to share our methods of resumption and admission algorithms in the post-epidemic period. It is not our intention to provide a solution that work worldwide, but we do offer one possible solution that we hope will be of help to inspire others around the world to plan for the resumption of their departments in the post-epidemic period.

Acknowledgments We would like to acknowledge Zhuangzhuang Miao and Wei Sun for their assistance in data collection. We would like to acknowledge John Seven for his assistance in manuscript revision.

Authors' contributions Conceptualization:Yiwei Qi, Ting Lei; Methodology: Yiwei Qi, Xueyan Wan, Liang Lu, Chunxia Qi, Ting Lei; Formal analysis and investigation: Yiwei Qi, Xiaojin Liu, Liang Lu, Feng Hu, Kai Zhao; Writing_-original draft preparation: Yiwei Qi, Xiaojin Liu, Xueyan Wan; Writing_-review and editing: Yiwei Qi, Kai Shu, Ting Lei; Revision: Yiwei Qi, Ting Lei; Resources: Kai Zhao, Chunxia Qi, Feng Hu, Kai shu, Ting Lei; Supervision: Kai Shu, Ting Lei.

All authors read and approved the final manuscript.
Data availability All data generated or analyzed during this study are included in this published article.

Code availability The statistics and graphs were performed using GraphPad Prism 7.0.

\section{Declarations}

Ethical approval This study was approved by the ethics committee of Tongji Hospital, Wuhan, China. All procedures were performed in accordance with the ethical standards of the institutional and/or national research committee and the 1964 Helsinki Declaration and its later amendments or comparable ethical standards.

Informed consent For this type of retrospective study, formal consent is not required.

Consent for publication (include appropriate statements).

Not applicable.

Conflicts of interest The authors report no conflict of interest concerning the materials or methods used in this study or the findings specified in this paper.

\section{References}

1. Bai Y, Yao L, Wei T, Tian F, Jin D-Y, Chen L, Wang M (2020) Presumed Asymptomatic Carrier Transmission of COVID-19. JAMA 323:1406. https://doi.org/10.1001/jama.2020.2565

2. Clark A, Jit M, Warren-Gash C, Guthrie B, Wang HHX, Mercer SW, Sanderson C, McKee M, Troeger C, Ong KL, Checchi F, Perel P, Joseph S, Gibbs HP, Banerjee A, Eggo RM (2020) Global, regional, and national estimates of the population at increased risk of severe COVID-19 due to underlying health conditions in 2020: a modelling study. Lancet Glob Heal 1-15. https://doi.org/ 10.1016/S2214-109X(20)30264-3

3. Fang Y, Zhang H, Xie J, Lin M, Ying L, Pang P, Ji W (2020) Sensitivity of Chest CT for COVID-19: Comparison to RT-PCR. Radiology 200432. https://doi.org/10.1148/radiol.2020200432

4. Fraser J Uncounted millions had COVID-19 symptoms, but no positive test. In: USA Today. https://www.usatoday.com/in-depth/ news/investigations/2020/07/17/covid-19-no-positive-test-uncou nted-millions-still-had-symptoms/5407855002/. Accessed 17 Jul 2020

5. Huang P What We Know About The Silent Spreaders Of COVID19. In: NPR. https://www.npr.org/sections/goatsandsoda/2020/ 04/13/831883560/can-a-coronavirus-patient-who-isnt-showingsymptoms-infect-others. Accessed 13 Apr 2020

6. Iorio-Morin C, Hodaie M, Sarica C, Dea N, Westwick HJ, Christie SD, McDonald PJ, Labidi M, Farmer J-P, Brisebois S, D’Aragon F, Carignan A, Fortin D (2020) Letter: The Risk of COVID-19 Infection During Neurosurgical Procedures: A Review of Severe Acute Respiratory Distress Syndrome Coronavirus 2 (SARSCoV-2) Modes of Transmission and Proposed NeurosurgerySpecific Measures for Mitigation. Neurosurgery 87:E178-E185. https://doi.org/10.1093/neuros/nyaa157

7. Kronbichler A, Kresse D, Yoon S, Lee KH, Effenberger M, Il SJ (2020) Asymptomatic patients as a source of COVID-19 infections: A systematic review and meta-analysis. Int J Infect Dis. https://doi.org/10.1016/j.ijid.2020.06.052

8. Mardani, Nadji SA, Aghazadeh Sarhangipor K, Sharifi-Razavi A, Baziborun M (2020) COVID-19 infection recurrence presented 
with meningoencephalitis. New Microbes New Infect 100732. https://doi.org/10.1016/j.nmni.2020.100732

9. Prokop M, van Everdingen W, van Rees Vellinga T, Quarles van Ufford J, Stöger L, Beenen L, Geurts B, Gietema H, Krdzalic J, Schaefer-Prokop C, van Ginneken B, Brink M (2020) CO-RADS - A categorical CT assessment scheme for patients with suspected COVID-19: definition and evaluation. Radiology 201473. https:// doi.org/10.1148/radiol.2020201473

10. Rosenbaum L Wuhan Lockdown Finally Ends After 76 Days; Officials Alert For Second Wave Of Infections. In: Forbes. https:// www.forbes.com/sites/leahrosenbaum/2020/04/07/wuhan-lockd own-finally-ends-after-76-days-officials-alert-for-second-waveof-infections/\#4e948f3b21e3. Accessed 7 Apr 2020

11. Shen N, Zhu Y, Wang X, Peng J, Liu W, Wang F, Lu Y, Cheng L, Sun Z (2020) Characteristics and diagnosis rate of 5630 subjects receiving SARS-CoV-2 nucleic acid tests from Wuhan, China. JCI insight 5:1-7. https://doi.org/10.1172/jci.insight.137662

12. Tan Y-T, Wang J-W, Zhao K, Han L, Zhang H-Q, Niu H-Q, Shu K, Lei T (2020) Preliminary Recommendations for Surgical Practice of Neurosurgery Department in the Central Epidemic Area of 2019 Coronavirus Infection. Curr Med Sci 40:281-284. https:// doi.org/10.1007/s11596-020-2173-5
13. Wei L, Lin J, Duan X, Huang W, Lu X, Zhou J, Zong Z (2020) Asymptomatic Patients Can Contaminate Their Surroundings an Environment Sampling Study. mSphere 5:3-6. https://doi.org/10. 1128/msphere.00442-20

14. Ye F, Xu S, Rong Z, Xu R, Liu X, Deng P, Liu H, Xu X (2020) Delivery of infection from asymptomatic carriers of COVID-19 in a familial cluster. Int J Infect Dis 94:133-138. https://doi.org/ 10.1016/j.ijid.2020.03.042

15. Zagrizek A (2020) Public transport resumption blamed for new COVID-19 cases. https://www.ynetnews.com/article/HyZcBIZnI. Accessed 31 May 2020

16. COVID-19 dashboard. Accessed 2020 July 7. In: Johns Hopkins Univ. Cent. Syst. Sci. Enginnering. https://www.arcgis.com/apps/ opsdashboard/index.html\#/bda7594740fd40299423467b48e9ec f6. Accessed 7 Jul 2020

Publisher's Note Springer Nature remains neutral with regard to jurisdictional claims in published maps and institutional affiliations. 